\section{ORIGINAL RESEARCH}

\section{S. Maimon}

E. Nossek

I. Strauss

D. Blumenthal

V. Frolov

Z. Ram

\title{
Transarterial Treatment with Onyx of Intracranial Dural Arteriovenous Fistula with Cortical Drainage in 17 Patients
}

BACKGROUND AND PURPOSE: Intracranial DAVFs with cortical venous drainage have a high tendency to bleed. Complete closure of these lesions is essential to prevent clinically deleterious events. We describe our experience using Onyx in an arterial approach for treatment of DAVFs in 17 patients.

MATERIALS AND METHODS: Between 2006 and 2010, we used Onyx for performing transarterial embolization in 17 patients with intracranial DAVFs and cortical venous drainage. Clinical assessment was performed before and after every treatment at discharge and at follow-up. Fourteen patients underwent follow-up MR imaging and MRA, 8 of them also underwent follow-up diagnostic angiography.

RESULTS: Fifteen patients (88\%) underwent 1 procedure. Complete obliteration by embolization with Onyx was achieved in 16 patients (94\% acute obliteration). The mean amount of Onyx injected was 2.3 $\mathrm{mL}$ (range, 0.4-4.8 mL). The sole technical complication was an embolus to a branch of the MCA, which was resolved by intra-arterial tPA injection. A clinical complication of transient trochlear nerve palsy in the same patient due to mass effect of Onyx resolved spontaneously within 3 months.

CoNCLUSIONS: Intra-arterial embolization of cranial DAVFs with cortical venous drainage by using Onyx results in a high rate of complete obliteration (94\%) with low morbidity (6\%). Follow-up DSA in 8 patients revealed no evidence of reopening.

ABBREVIATIONS CVD = cortical venous drainage; DAVF = dural arteriovenous fistula; DMSO = dimethyl-sulfoxide; $\mathrm{EVOH}=$ ethylene-vinyl alcohol copolymer; $n$-BCA = n-butyl 2-cyanoacrylate

T he 2 systems commonly used for categorizing DAVFs, the scales of Borden-Shucart ${ }^{1}$ and Cognard, ${ }^{2}$ rely entirely on angiographic features. The most critical anatomic feature is the identification of CVD: Borden-Shucart types II and III and Cognard types IIa and b, III, IV, and V.

This feature identifies lesions at high risk for future hemorrhage or ischemic neurologic injury. ${ }^{3}$ In type II, the venous drainage is from the fistula to a sinus and retrogradely to a cortical vein, while in type III, all drainage is directly to a pial vein, making type III riskier.

Onyx (ev3, Irvine, California) is a nonadhesive liquid embolic agent that is a mixture of EOVH, DMSO, and tantalum powder. It was evaluated at the University of California, Los Angeles Medical Center from January 1998 to May 1999 and was first used in Europe by Monayer et $\mathrm{al}^{4}$ for brain AVMs in January 1999. The US Food and Drug Administration approved the intravascular use of Onyx for embolization of intracranial AVMs in July 2005. Endovascular embolization with Onyx has evolved in the past few years as one of the tools for treating intracranial DAVFs. The ability to occlude malformations, which are fed by multiple vessels, and the relatively

Received February 27, 2011; accepted after revision April 25.

From the Department of Neurosurgery (S.M., E.N., I.S., D.B., V.F., Z.R.) and Interventional Neuroradiology Unit (M.S., V.F.), Tel Aviv Medical Center, Faculty of Medicine, Tel Aviv University, Tel Aviv, Israel.

Drs Maimon and Nossek contributed equally to this work.

Paper previously presented at: Anatomy-Biology-Clinical Correlation/Work Group in Interventional Neuroradiology meeting, January 16-21, 2011; Val d'Isère, France.

Please address correspondence to Shimon Maimon, MD, Department of Neurosurgery, Tel-Aviv Medical Center, 6 Weizman St, Tel Aviv 64239, Israel; e-mail: maimonsm@ zahav.net.il

http://dx.doi.org/10.3174/ajnr.A2728 long duration of injection allow good penetration of Onyx into the vascular malformation. ${ }^{5}$ After acquiring experience in using Onyx for AVM and tumor embolization, we substituted Onyx for glue in treating DAVFs as well.

We describe our results in using this new curative treatment strategy in Borden-Shucart ${ }^{1}$ types II and III fistulas, with special emphasis on the parameters of safety, feasibility, and efficacy.

\section{Materials and Methods}

\section{Patients}

Between October 2006 and June 2010, 19 patients were admitted to our department for treatment of intracranial DAVFs with suspected cortical venous drainage. One of the patients was diagnosed angiographically as a having type I DAVF (no cortical venous drainage) and, therefore, was not treated. A second patient was treated by a transvenous route due to anatomic considerations. The remaining 17 patients were treated transarterially with Onyx for curative purposes in a total of 20 embolization sessions. There were 15 (88.2\%) male and $2(11.8 \%)$ female patients; the mean age was 56 years (range, $22-71$ years). According to the Borden-Shucart classification system, ${ }^{1} 16$ patients in this group harbored type III DAVFs, and 1, a type II DAVF.

The presenting symptoms were recorded before the first treatment session (Table 1).

In most cases, MR imaging and MRA or high-quality CTA or both were used as diagnostic tools and for planning the treatment. We did not perform a pretreatment diagnostic angiography in a separate session. The diagnostic angiography was the first part of the embolization session, which we performed with the patient under general anesthesia. Follow-up clinical and imaging (angiography/MR imaging 


\begin{tabular}{lc}
\hline Table 1: Presenting symptoms & \\
\hline Main Presenting Symptom & No. of Patients \\
\hline Bleeding & 5 \\
Tinnitus & 3 \\
Trigeminal neuralgia & 4 \\
Ataxia & 1 \\
Seizures & 1 \\
Memory difficulties & 1 \\
Parkinsonism & 1 \\
Incidental & 1 \\
Total & 17
\end{tabular}

and MRA) data on these patients were recorded up to November 2010 and used for this report.

\section{Embolization Materials}

Onyx is composed of an EVOH dissolved in DMSO with tantalum powder (35\% weight/volume) added for visualization (radio-opacity). It is supplied in ready-to-use vials, each containing EVOH, DMSO, and tantalum powder in 3 concentrations, $6.0 \%, 6.5 \%$, and $8 \%$, corresponding to a viscosity of 18,20 , and $34 \mathrm{cP}$. With a lower concentration of the copolymer, the agent is less viscous and more distal penetration can be achieved. We used Onyx-18, the least viscous preparation, for all of our patients.

\section{Microcatheters}

We used 2 types of Onyx-compatible microcatheters: the Marathon (ev3) and the Sonic (Balt, Montmorency, France). The Sonic is a microcatheter with a detachable tip designed for prolonged Onyx injections in vascular lesions. According to the description in the insert of the manufacturer, the detachable tip is connected by glue that dissolves slowly on contact with the DMSO in the Onyx solution. At the end of a procedure using the Sonic, the tip is detached by gently pulling on the catheter, thus minimizing the risk of the microcatheter being entrapped, as well as the risk of vessel rupture. The distal part is left in the Onyx reflux cast. This feature of the microcatheter allows time for a relatively prolonged and gentle injection. ${ }^{6}$ The Sonic microcatheter comes in 3 forms: 1.5F 25-mm, 1.2F 15-mm, and 1.2F 25$\mathrm{mm}$ (the first number indicates the diameter of the tip and the second indicates the length of the detachable segment). The Marathon microcatheter is equally compatible with both Onyx and n-BCA injections. It is stiffer and can be advanced more easily than the Sonic in a slow-flow vessel and tortuous feeders where the microcatheter must be pushed with the aid of a guidewire. It has only 1 marker on its distal tip, while the Sonic microcatheter has 3 markers.

Navigation is usually performed with a guidewire in front in both microcatheters when used for the treatment of DAVFs, due to the fact that the feeding artery usually originates from the external carotid system. A guidewire is needed to advance the microcatheter toward the fistula. We have made every effort to get as close as possible to the lesion so as to prevent any unintended spread of Onyx to other vessels and thereby reduce the risk of peripheral cranial nerve ischemic deficit.

\section{Procedure}

All embolization procedures were performed with the patient under general anesthesia at the same center under the same conditions by the first author (S.M.) by using a monoplane Angio Suite (Philips Healthcare, Best, the Netherlands). Vascular access was obtained via a transfemoral approach by using a 6F MPC or MPD (Cordis/Johnson \& Johnson, Miami Lakes, Florida) guiding catheter. Treatment was performed immediately following diagnostic angiography to delineate the vascular architecture of the lesions.

Heparin, $1000 \mathrm{IU}$ per liter of saline, was only used in the flushing system of the guiding catheter. No subsequent intravenous heparin was administered during or after the procedure.

After the diagnostic stage, the microcatheter was navigated to a selected feeding artery as close as possible to the fistula. The catheter was then positioned to a location to achieve optimal Onyx-injection conditions and to enable visualization of the 2 proximal markers (Sonic) or the distal reflux (Marathon) during the injection.

Onyx injection was performed according to the plug-creating technique. The goal was to create a hard plug of Onyx around the distal part of the microcatheter. This creates a pseudo-wedge position, preventing further reflux of the embolizing material and enabling its penetration into the fistular compartments. This technique allows vascular connections to be opened between different compartments and to fill other feeders in a retrograde manner. The plug was created by small injections of Onyx and in-between periods of noninjection. These noninjection periods typically vary from a few seconds to 2 minutes and, according to our experience, are determined by different factors, such as vessel size, flow, length of the detachable zone, and position of the microcatheter tip relative to the fistula. We used a 2-minute cutoff because longer noninjection periods can result in occlusion of microcatheter tip.

Onyx was injected in a continuous manner or in pulses, depending on the stage of the treatment and the penetration rate. We have injected Onyx continuously if it showed good penetration. When the Onyx cast stopped advancing, we used small and short pulses of injection, taking special care to prevent excessive reflux around the distal part of the microcatheter. ${ }^{7}$

The primary goal of embolization of a DAVF is to fill the fistula itself and the proximal draining veins. A secondary goal (but not mandatory in all cases) is to retrogradely fill the other fistula feeders. We must occlude the proximal draining veins completely at the end of treatment. On the basis of our own experience and due to the fact that Onyx is a mechanical occlusion material and not thrombogenic (such as $n$-BCA), we overfilled the lesion and proximal draining veins. Before injecting the Onyx, we evaluated the venous draining anatomy and defined the sites on the venous side that needed to be occluded and the areas that we preferred the Onyx not enter and occlude. This is an important step in the treatment that can prevent any unwanted venous occlusion.

Injection of Onyx was disconnected when the lesion was considered obliterated. This was determined by the following criteria:

1) The proximal part of the draining veins (at least the first $2 \mathrm{~cm}$ ) is filled with an attenuated cast of Onyx

2) No early venous drainage is visualized.

When possible, retrograde occlusion with Onyx of the network of other feeders nearby the lesion is preferred.

\section{Results}

Seventeen patients with cranial DAVFs were treated transarterially with Onyx at our institution between October 2006 and August 2010. Twenty-seven arterial feeders were embolized with Onyx in 20 procedures (1.4 feeders/procedure). Fifteen patients underwent 1 treatment session, 1 patient had 2 sessions (for 2 nearby fistulas), and 1 patient had 3 sessions.

The patient who had 2 treatment sessions was treated in his 


\begin{tabular}{|c|c|c|c|}
\hline $\begin{array}{l}\text { Patient } \\
\text { No. }\end{array}$ & $\begin{array}{l}\text { No. of } \\
\text { Feeders }\end{array}$ & $\begin{array}{c}\text { No. of } \\
\text { Pedicles } \\
\text { Treated }\end{array}$ & $\begin{array}{c}\text { Time of Follow-Up } \\
\text { after Treatment }\end{array}$ \\
\hline 1 & 7 & 1 & $\mathrm{MRI} / \mathrm{A}, 12 \mathrm{mo}$ \\
\hline 2 & 10 & 2 & Angio, $16 \mathrm{mo}$ \\
\hline 3 & 6 & 1 & $\mathrm{MRI} / \mathrm{A}, 12 \mathrm{mo}$ \\
\hline 4 & 9 & 1 & $\mathrm{MRI} / \mathrm{A}, 11 \mathrm{mo}$ \\
\hline 5 & 12 & 1 & $\mathrm{MRI} / \mathrm{A}, 6 \mathrm{mo}$ \\
\hline 6 & 9 & 2 & Angio, $3 \mathrm{mo}$ \\
\hline 7 & 7 & 1 & Angio, 6 mo \\
\hline 8 & 8 & 2 & Angio, $12 \mathrm{mo}$ \\
\hline 9 & 10 & 2 & Angio, 4 mo \\
\hline 10 & 3 & 1 & $\mathrm{MRI} / \mathrm{A}, 10 \mathrm{mo}$ \\
\hline 11 & 8 & 1 & Angio, $5 \mathrm{mo}$ \\
\hline 12 & 9 & 3 & Not done yet \\
\hline 13 & 6 & 1 & $\mathrm{MRl} / \mathrm{A}, 8 \mathrm{mo}$ \\
\hline 14 & 3 & 1 & Angio, 4 mo \\
\hline 15 & 2 & 1 & Angio, 4 mo \\
\hline 16 & 7 & 2 & Not done yet \\
\hline 17 & 4 & 1 & Not done yet \\
\hline
\end{tabular}

Note:-MRI/A indicates MR imaging/MR angiography; Angio, angiography.

first session transarterially with Onyx. The second fistula nearby in the sigmoid-jugular junction was treated by a transvenous approach with coils, due to anatomic considerations.

Each fistula was closed by the end of each treatment session. The second session (treatment with coils) is not included in this study.

We used the Sonic microcatheter in 13 arterial feeders, the Marathon microcatheter in 11 feeders, and the Echelon (ev3) in 3 feeders. In 11 (64\%) cases, the fistulas were occluded from 1 feeder (Table 2). The mean amount of Onyx injected through a single feeder by using the Sonic microcatheter was $1.9 \mathrm{~mL}$ and, for the Marathon microcatheter, $1.3 \mathrm{~mL}$. Complete occlusion was achieved in 16 of 17 patients (94\%), and near-complete occlusion, in the remaining patient who underwent 3 sessions for a complex petrosal fistula. This patient experienced transient minor fourth nerve palsy and emboli to the MCA branch during the second treatment session. The nerve palsy was due to mass effect from the big Onyx cast (nearly $4 \mathrm{~mL}$ was injected) and resolved in a few months. The embolus to the MCA was dissolved immediately with selective intra-arterial rtPA with no neurologic sequelae.

In 15 patients, complete occlusion was achieved after a single treatment session. One patient with 2 adjacent fistulas underwent 2 treatment sessions; complete occlusion in the fistula with cortical drainage was achieved in the first session by transarterial Onyx injection. The second fistula was cured with coils by a transvenous route.

The patient who underwent 3 treatment sessions with near-complete occlusion was referred for open surgery during which the fistula was occluded. In this patient, the size and tortuosity of the feeder of the remnant, the petrosal branch of the middle meningeal artery, precluded endovascular complete obliteration.

\section{Follow-Up}

Clinical assessment was performed before and after every treatment at discharge and at follow-up. Fourteen patients underwent follow-up MR imaging and MRA between 3 and 12 months after treatment; 8 of them also underwent follow-up diagnostic angiography. Three patients have undergone clinical follow-up only and are waiting for follow-up imaging.

The patient with near-complete closure had a small residual fistula that did not change from the second session and could not be occluded in the third session. He later underwent open surgery for closure of that residual fistula without clinical complications.

Clinically all patients improved after treatment at the follow-up period, except for 1 patient who remained at his baseline neurologic condition.

In summary we experienced 1 technical complication (1/ $20-5 \%$ ) with no permanent neurologic deficit and 1 transient neurologic deficit (fourth nerve palsy), accounting for a clinical complication rate of 5.8\% (1/17).

There was no evidence of recurrence or changes in the treated fistulas in the group with follow-up angiography. There was a good correlation between the follow-up MR imaging and MRA, which were performed before the follow-up angiography, in 8 patients who underwent a posttreatment angiographic control study.

\section{Discussion}

The success of intra-arterial embolization as a definitive treatment of DAVFs before the introduction of Onyx was limited. ${ }^{8}$ The use of polyvinyl alcohol particles promotes proximal occlusion only, but permanent obliteration of the fistula is rare. To have an effect from $n$-BCA, extensive experience in both its preparation and delivery is required. ${ }^{9}$ Moreover the chemical and physical properties, such as polymerization rate, binding, and viscosity, can make the use of $n$-BCA unpredictable. ${ }^{9}$

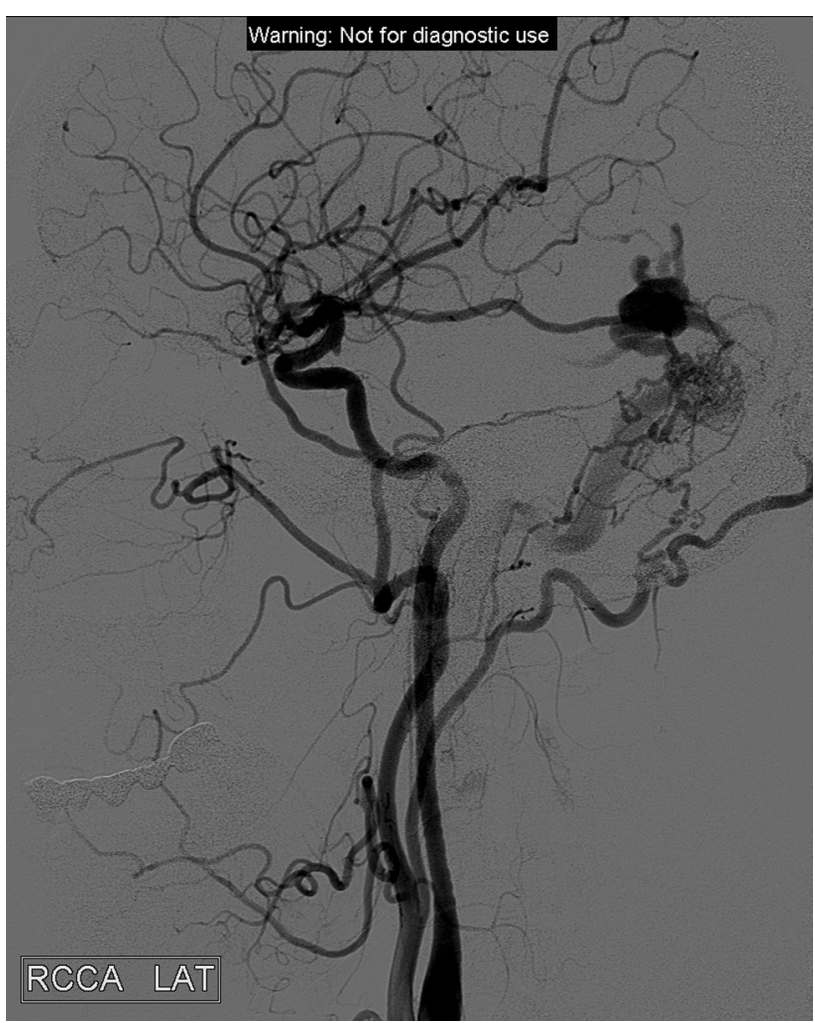

Fig 1. Left lateral common carotid artery. A DAVF fed mainly by middle meningeal branches draining to cortical veins with a varix. 

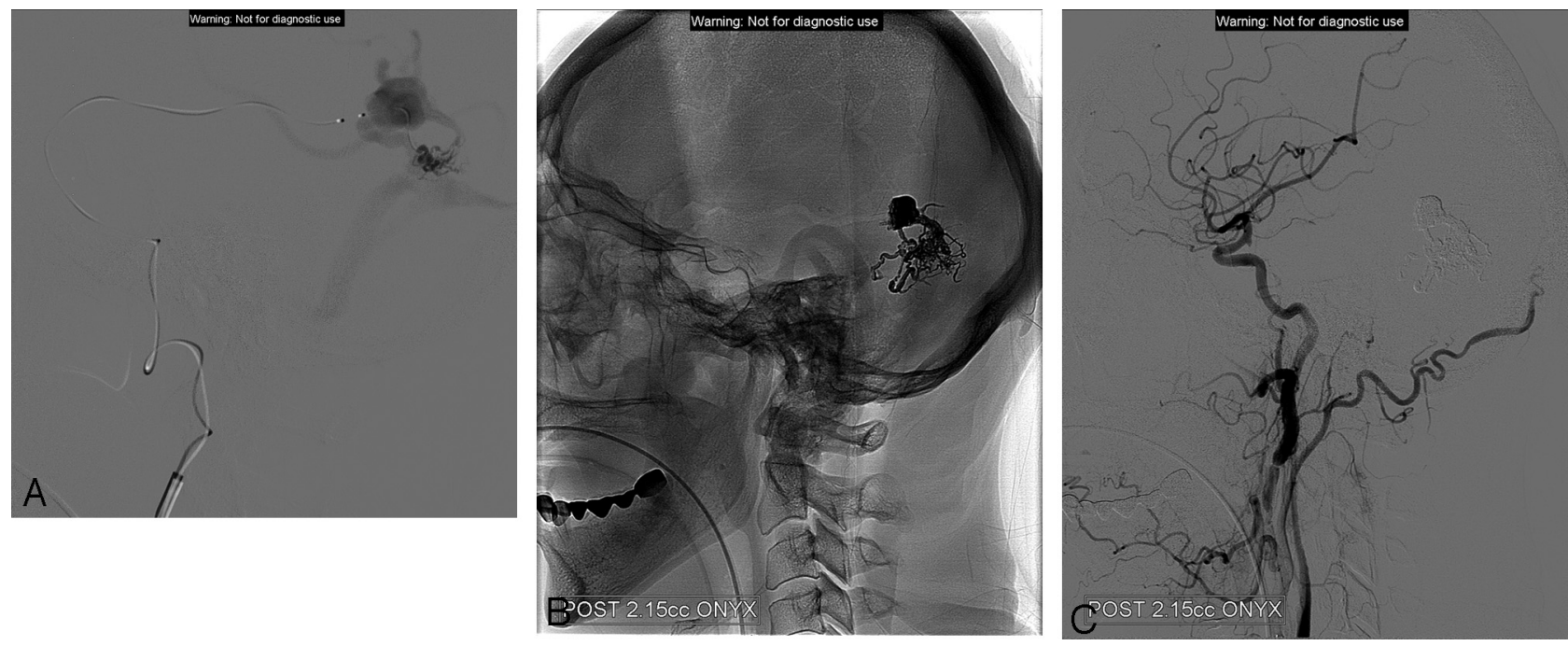

Fig 2. $A$, Superselective injection with the Sonic microcatheter. Image demonstrates the fistula and the pial cortical draining vein with the varix, draining to the vein of Labbe. $B$, The final cast includes the proximal part of draining vein and the network of feeders. $C$, The completion injection and full closure of the fistula.

Onyx is a new liquid embolic agent for the treatment of brain AVMs. Onyx is supplied in ready-to-use vials and is prepared in 3 different concentrations. With a lower concentration of the copolymer, the agent is less viscous and more distal penetration can be achieved. Most of the clinical experience with Onyx had originated from the treatment of brain AVMs. Recent published series report high occlusion rates, with $20 \%-$ 49\% of brain AVMs completely occluded either solely with Onyx or in combination with $n$-BCA. According to the literature, there are several advantages to using Onyx for DAVF treatment: First, the reflux re-injection technique (as described in an earlier publication $)^{7}$; second, the prolonged injection time; third, the more predictable effect; and finally, excellent penetration into the fistula network. ${ }^{8-10}$ Those features permit slow material injection with a good control of spreading of the Onyx in the lesion and the nearby area.

To our knowledge, the literature concerning the use of Onyx for DAVF treatment is sparse. One case report described a cavernous carotid fistula treated by a combination of coiling and embolization with Onyx. ${ }^{10}$ Another case report described the treatment of a fistula in the region of the lesser sphenoid wing. ${ }^{9}$ An additional report described treatment of a fistula of the superior sagittal sinus. ${ }^{8}$ In all the reported cases, the lesions were successfully treated with no need for further treatment (surgery or radiosurgery).

Toulgoat et $\mathrm{al}^{11}$ reported a series of 6 patients with intracranial DAVFs who were treated by Onyx. Treatments resulted in complete anatomic occlusion of the malformations. The Onyx filled all malformations and their draining vessels after a single arterial feeder catheterization.

Carlson et $\mathrm{al}^{12}$ reported a series of 6 patients; complete occlusion was achieved in 5 patients $(83.3 \%)$. None of their patients had worsening of neurologic function. One patient had a complication of a retained catheter fragment.

Another series of 12 patients was reported by Nogueira et al. ${ }^{13}$ Ten patients had complete resolution on an immediate postprocedure angiography. One patient had recurrence on follow-up angiography. There was neither reported morbidity nor mortality.
Cognard et $\mathrm{al}^{14}$ enrolled 30 patients with DAVFs in a prospective study. Five patients were previously embolized by other materials. Twenty-four patients had complete resolution (20 in a single session). There were 2 reported complications: first, intracerebral bleeding due to draining vein thrombosis; and second, cranial nerve (third and forth) palsy, from which the patient did not recover.

There are dural fistulas in which the arterial approach cannot be used due to multiple tortuous feeders making the navigation and glue injection technically difficult. Treatment of such cases can result in failure to get a complete occlusion due to the inability of the embolizing material to reach and occlude the venous part of the lesion.

The decision regarding which material to use and the preferred route to treat a lesion depends on the anatomy, specifically location, feeder size and tortuosity, and proximity to vital brain structures.

According to our experience with Onyx in the treatment of $>100$ patients with AVMs in the past 4 years, we learned how to manage and occlude fistulas inside the network of the AVM with Onyx. ${ }^{7}$ As was previously reported in the literature, we also noted the superior penetration ability and the cast formation of the material, the ability to approach the proximal part of the draining veins, and the occlusion of other feeders retrogradely while injecting from the 1 catheterized feeder. All these capabilities of Onyx, along with the prolonged injection time with a detachable-tip microcatheter and the option to perform diagnostic angiography at the time of the treatment, influenced our decision to use it in the treatment of dural fistulas. We were able to occlude complicated fistulas with multiple feeding vessels in 1 session in most of our patients.

In all cases, an Onyx-compatible microcatheter was advanced to an arterial feeder in proximity to the fistula after analyzing the architecture of the vessels, including the draining veins, because knowledge of the venous anatomy is most important for a safe and definitive treatment. For curing the lesion, Onyx casts must include the proximal part of the draining vein.

There are situations in which the transvenous route is the 
preferred choice. This is when no good feeders to the lesion can be found or the occlusion of the feeders can lead to cranial nerve deficit (as we anticipated in 2 other cases).

This series does not include a dural cavernous sinus fistula. In such cases, if feasible, we prefer the transvenous route.

Diagnostic angiography is the optimal follow-up procedure. We did not observe any lesion changes in those who underwent postprocedural angiography. It is not clear if a patient with a full Onyx cast of the lesion, including the proximal veins, with no early venous drainage at end of treatment, requires follow-up angiography. Some patients refused angiography and asked for MR imaging and MRA (with gadolinium) follow-up only. The angiography findings correlated well in all cases with the MR imaging and MRA findings in our cohort, but the group of patients that underwent angiography as well as MR imaging/MRA is too small to reach definitive conclusions about follow-up only with MR imaging and 3D time-offlight MRA. It shows encouraging results but needs further case evaluation.

We still encourage our patients to undergo follow-up diagnostic angiography, as the preferred tool to evaluate the treatment.

\section{Conclusions}

By using Onyx in the transarterial route in the treatment of DAVFs with cortical drainage, we can fill the fistula and, with a long duration of injection, can achieve optimal penetration and a good cast formation in both feeders and draining veins. Only when we fill the venous side of the fistula more than a few centimeters, can we be sure it is fully occluded. The properties of Onyx make it a good tool for obliteration of complex DAVFs from an arterial side with low morbidity. Transarterial endovascular treatment with Onyx is currently our first and preferable treatment option for obliteration of cranial DAVFs with cortical venous drainage.

\section{Acknowledgments}

We thank Esther Eshkol for editorial assistance.

\section{References}

1. Borden JA, Wu JK, Shucart WA. A proposed classification for spinal and cranial dural arteriovenous fistulous malformations and implications for treatment. J Neurosurg 1995;82:166-79

2. Cognard C, Gobin YP, Pierot L, et al. Cerebral dural arteriovenous fistulas: clinical and angiographic correlation with a revised classification of venous drainage. Radiology 1995;194:671-80

3. Weber W, Kis B, Siekmann R, et al. Endovascular treatment of intracrania arteriovenous malformation with Onyx: technical aspects. AJNR Am J Neuroradiol 2007;28:371-77

4. Monayer C. Hammami N. Piotin M. Nidal embolization of brain arteriovenous malformation using Onyx in 94 patients. AJNR Am J Neuroradiol 2007;28:518-23

5. van Rooij WJ. Sluzewski M, Beute GN. Brain AVM embolization with Onyx. AJNR Am J Neuroradiol 2007;28:172-77

6. Jahan R, Murayama Y, Gobin Y, et al. Embolization of arteriovenous malformation with onyx: clinicopathological experience in 23 patients. Neurosurgery. 2001;48:984-95

7. Maimon S, Strauss I, Frolov V, et al. Brain arteriovenous malformation treatment using a combination of Onyx and a new detachable tip microcatheter, SONIC: short-term results. AJNR Am J Neuroradiol 2010;31:947-54. Epub 2010 Feb 25

8. Arat A. Inci S. Treatment of a superior sagittal sinus dural arteriovenous fistula with Onyx: technical case report. Neurosurgery 2006;56:169-70

9. Rezende MT, Piotin M, Mouayer C, et al. Dural arteriovenous fistula of the lesser sphenoid wing region treated with Onyx: technical note. Neuroradiology 2006;48:130-34

10. Suzuki S, Lee DW, Jahan R, et al. Transvenous treatment of spontaneous dural carotid-cavernous fistulas using a combination of detachable coils and Onyx. AJNR Am J Neuroradiol 2006;27:1346-49

11. Toulgoat F, Mounayer C, Túlio Salles Rezende M, et al. Transarterial embolisation of intracranial dural arteriovenous malformations with ethylene vinyl alcohol copolymer (Onyx 18) [in French]. J Neuroradiol 2006;33:105-14

12. Carlson AP, Taylor CA. Yonas H. Treatment of dural arteriovenous fistula using ethylene vinyl alcohol (Onyx) arterial embolization as the primary modality: short-term results. J Neurosurg 2007;107:1120-25

13. Nogueira RG, Dabus G, Rabinov JD., et al. Preliminary experience with Onyx embolization for the treatment of intracranial dural arteriovenous fistulas. AJNR Am J Neuroradiol 2008;29:91-97

14. Cognard C, Januel AC, Silva NA Jr, et al. Endovascular treatment of intracranial arteriovenous fistulas with cortical venous drainage: new management using Onyx. AJNR Am J Neuroradiol 2008;29:235-41 